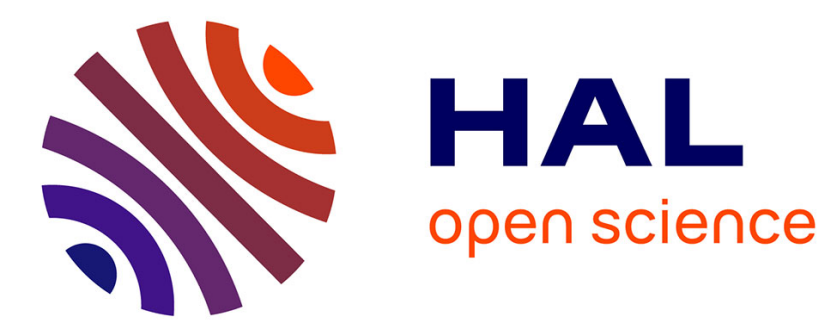

\title{
Synthèse du bruit rayonné par une machine tournante carénée
}

\author{
M. Jacob, M. Roger
}

\section{To cite this version:}

M. Jacob, M. Roger. Synthèse du bruit rayonné par une machine tournante carénée. Journal de Physique IV Proceedings, 1994, 04 (C5), pp.C5-981-C5-984. 10.1051/jp4:19945215 . jpa-00252900

\section{HAL Id: jpa-00252900 https://hal.science/jpa-00252900}

Submitted on 1 Jan 1994

HAL is a multi-disciplinary open access archive for the deposit and dissemination of scientific research documents, whether they are published or not. The documents may come from teaching and research institutions in France or abroad, or from public or private research centers.
L'archive ouverte pluridisciplinaire HAL, est destinée au dépôt et à la diffusion de documents scientifiques de niveau recherche, publiés ou non, émanant des établissements d'enseignement et de recherche français ou étrangers, des laboratoires publics ou privés. 


\title{
Synthèse du bruit rayonné par une machine tournante carénée
}

\author{
M.C. JACOB et M. ROGER
}

Laboratoire de Mécanique des Fluides et d'Acoustique, URA 263 du CNRS, Ecole Centrale de Lyon, UCB-Lyon I, BP. 163, 69131 Ecully cedex, France

\begin{abstract}
résumé: Une méthode de calcul du bruit rayonné par une machine tournante ayant un carénage court est présentée. Les sources aéroacoustiques sont obtenues à l'aide d'un code de calcul des charges instationnaires sur les pales. L'influence du carénage sur la propagation est calculée par une méthode intégrale d'éléments finis de surface. Une application montre l'effet de masque du carénage sur la propagation du son émis par la source.
\end{abstract}

abstract : A tool for predicting the acoustic far field of a fan in a short duct is presented. The aeroacoustic sources are obtained from a numerical calculation of the unsteady blade loadings. The diffraction of the free field by the duct is calculated with an integral boundary finite element method.An example illustrates the masking effect of a short duct on a rotor free field.

\section{INTRODUCTION}

Pour étudier le rayonnement acoustique d'une machine tournante carénée, l'approche classique consiste d'une part à déterminer les sources aéroacoustiques en fonction des paramètres cinématiques et géométriques de la machine, et d'autre part à prendre en compte l'influence du carénage sur le rayonnement des sources. Le premier aspect est appréhendé à l'aide des théories de l'aéroacoustique qui permettent d'exprimer les sources à partir des charges aérodynamiques instationnaires s'exerçant sur les pales de la machine[1]. Le deuxième aspect a pu faire l'objet d'investigations analytiques dans le cas de machines pourvues d'un carénage long telles que les turbomachines de l'aéronautique ou certains ventilateurs industriels : en effet, dans ce cas le rayonnement complet a pu êrre calculé en adaptant des théories modales développées pour des cylindres infiniment longs à des conduits semi-infinis [2],[3], puis à des cylindres finis d'allongement $L / D>>1$ [4]. Ces méthodes ne sont pas applicables à des écrans courts (L/D 1 ou L/D $<<1$ ) ou non cylindriques. Or, différents types de machines tournantes à carénage court ont été développés durant ces dernières années (propulseurs de sous-marins, rotors anti-couple de certains hélicoptères (fenestron), hélices carénées...).

C'est pour étudier leur rayonnement acoustique, que nous avons mis au point une approche globale qui combine un code de calcul de charges aérodynamiques instationnaires[5] avec un code de calcul de diffraction [6]. Cette approche ainsi que des premiers résultats sont présentés ici. Dans la section 2, nous décrivons succintement le champ libre d'un rotor situé en amont d'un obstacle fixe (interaction potentielle[7]). Dans la section 3, nous présentons l'approche numérique permettant d'établir le rôle diffractant du carénage. Dans la section 4, nous illustrons la méthode par un exemple de calcul complet et nous apportons les conclusions et perspectives de cette approche.

\section{BRUIT D'ORIGINE AERODYNAMIQUE D'UNE MACHINE TOURNANTE}

En raison de la périodicité du mouvement, le spectre du bruit d'une machine tournante est dominé par une série de raies aux fréquences multiples de la fréquence de passage des pales, chacune de ces raies pouvant 
à son tour être décomposée en une superposition de structures équivalentes à des modes tournants, dont le potentiel, à un rayon source fixé s'exprime par :

$$
\phi_{L, n}^{(m)}(M)=\int_{0}^{2 \pi} A(\alpha) \frac{e^{i\left(k r_{\alpha}+n \alpha-\omega t\right)}}{r_{\alpha}} d \alpha
$$

où :

$-\omega=m B \Omega$,

- B : nombre de pales du rotor,

$-\Omega:$ vitesse angulaire du rotor,

- $\mathrm{m}$ : numéro de la raie,

- $\mathrm{n}$ : numéro du mode qui dépend de $\mathrm{mB}$ et du type de source considéré,

- $r_{\alpha}$ : distance entre la source située à l'angle $\alpha$ et le point d'écoute $M$, de charge.

- $\mathrm{A}(\alpha)$ : terme d'amplitude et de directivité qui dépend de la géométrie du rotor et des harmoniques

Il est donc possible de calculer le bruit de chaque raie du spectre en étudiant séparément chacun des modes la constituant. L'avantage réside dans le fait :

- qu'un mode tournant est lui-même une répartition de sources ponctuelles déphasées dont le comportement acoustique est connu et qui peut être aisément implanté dans un code de calcul de diffraction.

- que seul un nombre limité de modes contribuent de façon significative à une raie donnée,

- que la structure d'un mode tournant est indépendante de la valeur exacte des charges aérodynamiques instationnaires, qui n'intervient que comme pondération .

Le rayonnement de modes tournants isolés placés ou non dans un conduit court, a été étudié par ailleurs $[6],[8],[9]$ et $[10]$.

\section{INFLUENCE DU CARENAGE}

L'influence du carénage sur la propagation du son émis par les sources est prise en compte en résolvant le problème de diffraction correspondant :

$$
\left\{\begin{array}{l}
\left(\Delta+k^{2}\right) \phi_{n}^{(m)}=b_{n}^{(m)} \\
\partial_{n_{p}} \phi_{n}^{(m)}(p)=0 \text { sur }(S)
\end{array}\right.
$$

où :

- $k$ est le nombre d'onde à la fréquence considérée $m B \Omega / 2 \pi$.

- $b_{n}^{(m)}$ est la distribution de monopôles équivalant au $n$-ième mode contribuant à la m-ième raie,

- $\phi_{n}^{(m)}$ le champ acoustique total correspondant,

- $\partial_{n_{p}}$ est la dérivée suivant la normale en un point $\mathrm{p}$ de la surface (S) du carénage,

La surface $(\mathrm{S})$ du carénage est considérée comme rigide vis-à-vis des ondes acoustiques, ce qui modélise bien son comportement diffractant dans la plupart des applications.

Ce problème peut être reformulé sous une forme intégrale équivalente :

$$
\left\{\begin{array}{l}
\phi_{n}^{(m)}(M)=\phi_{L, n}^{(m)}(M)-\int_{(S)} v(q) \partial_{n_{q}} G_{k}(M, q) d q \\
\partial_{n_{p}} \phi_{L, n}^{(m)}(p)=P . F \cdot \int_{(S)} v(q) \partial_{n_{p}} \partial_{n_{q}} G_{k}(p, q) d q \quad \text { sur }(S)
\end{array}\right.
$$


où :

$-G_{k}\left(M, M^{\prime}\right)=-\frac{e^{i k M M^{\prime}}}{4 \pi M M^{\prime}} \quad$ (6) est la fonction de Green en espace libre,

- P.F. désigne la partie finie de l'intégrale au sens de Hadamard,

- $v$ la densité de double couche sur la surface $(S)$,

- $\phi_{L, n}^{(m)}(M)=\int_{\mathfrak{R}^{3}} b_{n}^{(m)}\left(M^{\prime}\right) G_{k}\left(M, M^{\prime}\right) d M^{\prime} \quad$ (7). est le potentiel acoustique du son émis par les sources en champ libre.

Pour calculer $\phi_{n}^{(m)}$, on détermine d'abord $v$ en résolvant l'équation aux limites, puis on obtient $\phi_{n}^{(m)}$ à partir d'un calcul direct de $\phi_{L, n}^{(m)}$ et de l'intégrale de l'équation (4). [11].

Lorsque le carénage est d'épaisseur non négligeable cette méthode ne fournit pas la solution aux fréquences de résonance d'une cavité qui aurait la même surface que le carénage mais qui serait creuse. Pour pallier cette difficulté mathématique, on peut introduire dans la cavité un écran absorbant dont le rôle est d'amortir ces résonances [12].

La résolution proprement dite est effectuée par une méthode d'éléments finis de surface associée à une formulation variationnelle des équations intégrales précédentes [13]. La mise au point et la validation du code de calcul correspondant sont décrites dans la référence [6].

\section{EXEMPLE DE SYNTHESE DE BRUIT D'UNE MACHINE TOURNANTE CARENEE}

L'application proposée ici concerne les interactions potentielles entre un obstacle aval et un rotor de $B=9$ pales de rayon $\mathrm{Rs}=0,40 \mathrm{~m}$. Les harmoniques de charge sont évalués par le code de calcul aérodynamique pour les paramètres cinématiques et géométriques suivants :

- vitesse angulaire du rotor $\Omega=140 . \pi \mathrm{rad} / \mathrm{s}$,

- inclinaison des pales $\gamma=37^{\circ}$,

- nombre de Mach de rotation $\mathrm{Mr}=0,5$,

- fréquence de la lère raie : $\omega / 2 \pi=630 \mathrm{~Hz}$.

On montre que le potentiel du champ libre d'une raie peut s'écrire :

$$
\phi_{L}^{(m)}=\sum_{\substack{s=-\infty \\ n=m B-s}}^{+\infty} \int_{0}^{2 \pi} \phi_{L, n, \alpha}^{(m)}(M) d \alpha
$$

avec :

$-\phi_{L, n, \alpha}^{(m)}(M)=\frac{e^{i\left(k r_{\alpha}+n \alpha-\omega t\right)}}{4 \pi r_{\alpha}}\left[C_{1} \cos \theta+C_{2} \sin \theta \sin (\alpha-\varphi)\right]$

- $\mathrm{C}_{1}=-\mathrm{B} \mathrm{F}_{\mathrm{S}} \cos (\gamma) / 2 \pi \rho_{0} \mathrm{c}_{0}$,

$-\mathrm{C}_{2}=-\mathrm{B} \mathrm{F}_{\mathrm{S}} \sin (\gamma) / 2 \pi \rho_{0} \mathrm{c}_{0}$,

- $\rho_{0}$ et $c_{0}$ sont respectivement la densité et la célérité du son dans le milieu au repos.

Seuls les harmoniques de charge $F_{S}$ avec $m B\left(1-M_{\Gamma}|\sin \theta|\right) \leq s \leq m B\left(1+M_{\Gamma}|\sin \theta|\right)$, apportent une contribution non négligeable à la somme infinie.

Cette expression du champ libre permet d'obtenir une expression analytique de son gradient normal à $(S)$ qui intervient dans le calcul de diffraction.

Ce rotor est placé au centre d'un conduit cylindrique mince de diamètre $D=0,9 \mathrm{~m}$ et de longueur $\mathrm{L}=0,4 \mathrm{~m}$ discrétisé par 80 triangles de taille inférieure au quart de la longueur d'onde. La figure 2 montre le champ lointain de la première raie du spectre avec ou sans prise en compte du carénage. Seuls les modes $n=-4$ à 4 excités par les harmoniques $F s=5$ à 13 , contribuent de façon notable au niveau de 
cette raie.

On peut constater que pour cette raie, le carénage exerce globalement un effet de masque dans le plan du rotor, alors que le rayonnement est amplifié dans les directions proches de son axe. Cet effet n'était pas du tout prévisible car pour la fréquence considérée, la longueur d'onde est du même ordre de grandeur que les dimensions du carénage, si bien que celui-ci agit comme un écran diffractant sur le champ libre. De plus, ce résultat est confirmé par des observations expérimentales faites sur des machines réelles (réduction du bruit sous trace d'hélicoptères équipés d'un fenestron au lieu d'un rotor anti-couple non caréné).

Afin de conforter ce dernier point, nous envisageons d'appliquer notre outil au cas d'un fenestron d'hélicoptère. Cela nécessitera d'étendre ce protocole de synthèse de bruit à des écrans épais, qui n'ont été étudiés que pour des sources plus simples jusqu'ici.

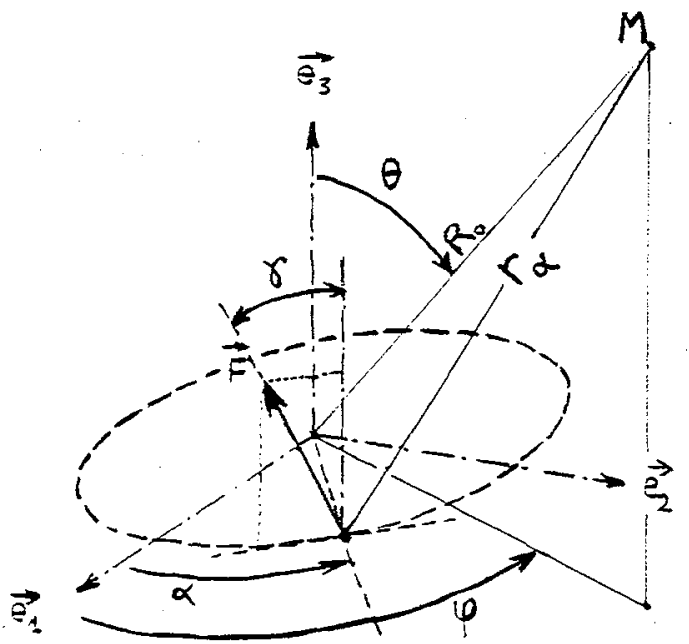

Fig. 1 paramètres géométriques

(le carénage d'axe $e_{3}$ n'est pas représenté)

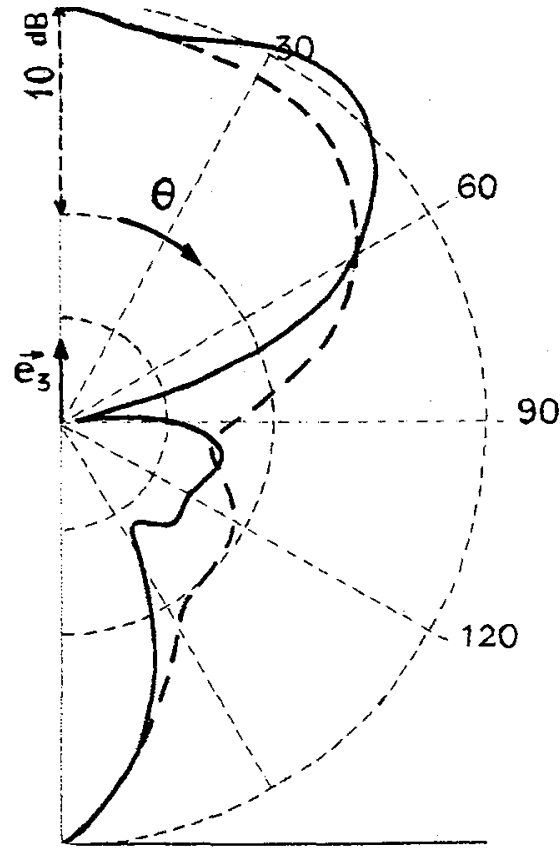

180

Fig. 2 : Directivité en champ lointain $(\mathrm{m}=1)$ : champ libre; champ total

\section{BIBLIOGRAPHIE}

[1] Ffwocs Williams J.E. and Hawkings D.L., Phil. Trans. Roy. Soc. A 264, (1969)321-342

[2] Levine H. and Schwinger, Phys. Rev. 73(4),(1948)

[3] Lansing D.L., NASA SP-228, (1970)

[4] Ogimoto K., University of Toronto, Institute for Aerospace studies, Report n $231,(1980)$

[5] Fournier F., Ecole Centrale de Lyon, Thèse de Doctorat n ${ }^{\circ} 88-09,(1988)$

[6] Jacob, M.C., Ecole Centrale de Lyon, Thèse de Doctorat $n^{\circ}$ 91-08, (1988)

[7] Fournier F. et Roger M. : C.R.A.S. 308(série II), (1989) 703-706

[8] Roger M., Jacob M.C. et Roland P., Contrat DRET-METRAFLU, Rapport final n³224 (1989).

[9] Roger M. Fournier F. and Jacob M.C., Contrat DRET-METRAFLU, Rapport final n ${ }^{\circ}$ 86-515 (1989).

[10] Jacob M.C.,"Diffraction de modes tournants par un écran axisymétrique - Etude théorique et expérimentale",, Journal de Physique supplément C2 (1990) 1177-1180

[11] Filippi P., J. Sound Vib. , 54(4) (1977) 473-500.

[12] Mebarek L. et Hamdi M., "Résolution du problème des fréquences irrégulières en équations intégrales", Xème Colloque d'Acoustique Aéronautique et Navale, Marseille, 19-21 novembre 1986.

[13] Hamdi M., UTC Compiègne, Thèse de Doctorat d'Etat,(1982). 